\title{
FEMTOSECOND LASER-ELECTRON INTERACTION IN A STORAGE RING STUDIED BY TERAHERTZ RADIATION *
}

\author{
K. Holldack ${ }^{\dagger}$, S. Khan, T. Quast, BESSY, 12489 Berlin, Germany, \\ R. Mitzner, Universität Münster, 48149, Münster, Germany
}

\begin{abstract}
The laser-induced energy modulation of relativistic electrons in the BESSY II storage ring was studied by temporal and spectral characterization of femtosecond far-infrared (THz) pulses being emitted after the electons have passed dispersive elements. The length of the femtosecond density modulation and its temporal decay have been measured as well as bunch shapes down to a length of $3 \mathrm{ps}$ (rms). The $\mathrm{THz}$ diagnostics is crucial for the operation of the recently commissioned "femtoslicing" source at BESSY.
\end{abstract}

\section{INTRODUCTION}

Femtosecond $\mathrm{x}$-rays pulses promise new insights into ultrafast physics in matter. With FEL sources being not yet available in the photon energy range above $100 \mathrm{eV}$ up to several keV, femtoslicing in storage rings may produce first results in that direction, albeit at much less intensity. The method of femtoslicing in a storage ring was pioneered by the ALS in Berkeley [1] demonstrating the generation of 300 fs hard x-rays from a dipole source. A new undulatorbased source providing sub-100 fs soft x-ray pulses from a helical undulator of excellent signal-to-background ratio has been successfully commissioned at BESSY II [2]. One of the key methods to start and operate this source has been the Terahertz (THz) diagnostics which is based on theoretical predictions on infrared emission in [1]. Being encouraged by detecting very tiny density modulations on short bunches at BESSY [3] a dedicated $\mathrm{THz}$ beamline was designed and constructed. First results have been reported in [4] and [5]. The present paper demonstrates that the $\mathrm{THz}$ emission is not only a reliable indicator with high dynamic range for laser-electron overlap [6] but yields additional information beyond.

\section{FEMTOSLICING AND THZ EMISSION}

The BESSY femtoslicing scheme is depicted in Fig.1. Electrons travelling with $1.7 \mathrm{GeV}$ kinetic energy copropagate with a laser pulse from a Ti:sapphire laser system of maximum pulse energy of $2.8 \mathrm{~mJ}$ at $1 \mathrm{kHz}$ repitition rate and $\geq 30 \mathrm{fs}$ fwhm pulse length at $800 \mathrm{~nm}$ in an undulator of $139 \mathrm{~mm}$ period length and 10 periods ("modulator") causing an oscillatory energy modulation. The off-energy electrons are transversly displaced in order to extract the short component of radiation emitted in the subsequent undulator UE56 (the "radiator"). A detailed description of the

\footnotetext{
* Funded by The Bundesminister für Bildung und Forschung and by the Land Berlin

† corresponding author, e-mail:holldack@bessy.de
}

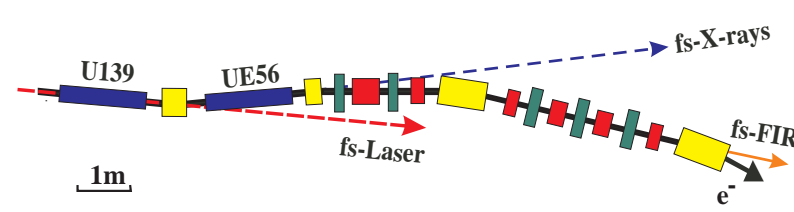

Figure 1: Schematic drawing of femtoslicing at BESSY showing modulator and radiator (blue), dipoles (yellow) and focussing optics (green and red). The emission of femtosecond laser-, $\mathrm{x}$-ray and FIR (THz)-pulses is indicated by arrows.

design of the BESSY angular separation scheme is given in [7]. Tracking simulations of electrons through the optical elements in Fig. 1 indicate that femtosecond density modulations on the bunch occur at all dipoles (yellow) downstream of the UE56. Although the structure is much shorter at the second dipole an easy geometric access to the source point was only possible at the third dipole, where an expected longitudional density modulation of the order of 0.1 $\mathrm{mm}$ length should give rise to coherent synchrotron radiation (CSR). Seeking for a reliable indicator for the laser electron interaction in the modulator, the basic idea to use CSR was that the coherent spectral power density:

$$
P_{c o h} \approx\left(N_{0} \frac{\sigma_{L}}{\sigma_{0}}\right)^{2} p(\omega)|F(\omega)|^{2}
$$

of such a longitudinal density modulation caused by a laser of length $\sigma_{L}$ on a bunch of $N_{0}$ electrons may exceed the spectral background power $P_{\text {inc }}=N_{0} p(\omega)$ from the regular bunch of length $\sigma_{0} \approx 50$ ps by orders of magnitude and can even exceed the incoherent power of up to 400 bunches in a multibunch train. Here, $|F(\omega)|$ is the magnitude of the Fourier transform of the longitudinal density modulation of electrons at the source point (far field). The quantity $p(\omega)$ means the incoherent power emitted by a single electron. This signal has been routinely used for optimizing the laser electron interaction in the U139 with respect maximum transverse overlap, the spectral overlap as well as the temporal synchronization. In all three cases, a maximum $\mathrm{THz}$ signal means also a maximum energy modulation between laser and electrons. The $\mathrm{THz}$ signal can be easily detected using standard far-infrared detectors either by a spectrum analyzer, an oscilloscope or a lock-in amplifier. Background sub- $\mathrm{THz}$ radiation due to the microbunching instability $\geq 4.5 \mathrm{~mA} /$ bunch is suppressed by using higher harmonics of the laser repetition frequency of $1 \mathrm{kHz}$ (i.e. the 27th harmonics) employing the fact that bursting CSR starts with a quasi-periodicity at lower fre- 
quencies. Another way to do this is to use a high pass filter $\geq 10 \mathrm{~cm}^{-1}$ discriminating the sub-THz spectrum of bursting CSR. An example of a single shot $\mathrm{THz}$ oscilloscope record measured with an InSb detector for femtoslicing in a regular user mode of BESSY II at $223 \mathrm{~mA}$ multibunch current and slicing on an extra bunch of $4 \mathrm{~mA}$ bunch current is plotted in the inset of Fig.2. A typical shot-to-shot stability of $5 \%$ at $\mathrm{THz}$ pulse energies up to $0.5 \mathrm{~nJ} /$ pulse is observed. However, varying the laser pulse length, a local minimum in the total $\mathrm{THz}$ pulse intensity was observed for highest energy modulation. Therefore, the $\mathrm{THz}$ emission was studied in more detail.

\section{$\mathrm{THz}$ afterglow and chirp control}

The analysis of the $\mathrm{THz}$ signal with high time resolution, as limited by the rise time of the detector of $0.3 \mu \mathrm{s}$ $(-3 \mathrm{~dB})$, is plotted in Fig. 2 showing one of the pulses in the inset. The density modulation caused by the laser shows an "afterglow" lasting up to the 15th turn in the ring. The first turn is detected with lower efficiency because the spectral response function of the InSb detector ranging from 50 $\mathrm{GHz}$ to $2 \mathrm{THz}$ favours long wavelengths from the following turns. A simple spectral characterization as depicted in



Figure 2: A THz pulse versus turn number detected using an InSb detector and a single shot $\mathrm{THz}$ time trace showing subsequent laser shots (inset).

Fig. 3 proves that a high frequency radiation is emitted only at the first turn. This fact was confirmed by further spectral measurements using signals gated on the individual turns and at the regular infrared beamline after $1 / 2$ the ring circumference [8]. As expected, the inital densitiy modulation is stretched by the non-isochronicity of the ring. The path length differences $\Delta L$ along the magnetic lattice for the laser energy modulated electrons increase turn by turn about a factor of 10 faster than for the electrons having only the natural energy spread. In first order they change according to $\Delta L=L_{0} \alpha \frac{\Delta p}{p}$ [9] where $\frac{\Delta p}{p}$ is the momentum change of the electrons, $\alpha=7.510^{-4}$ is the momentum compaction factor and $L_{0}$ is the ring circumference. The result is, that the temporal decay of the longitudinal density modulation and its power spectrum sensitively depend on the inital momentum transfer from the laser. This fact was

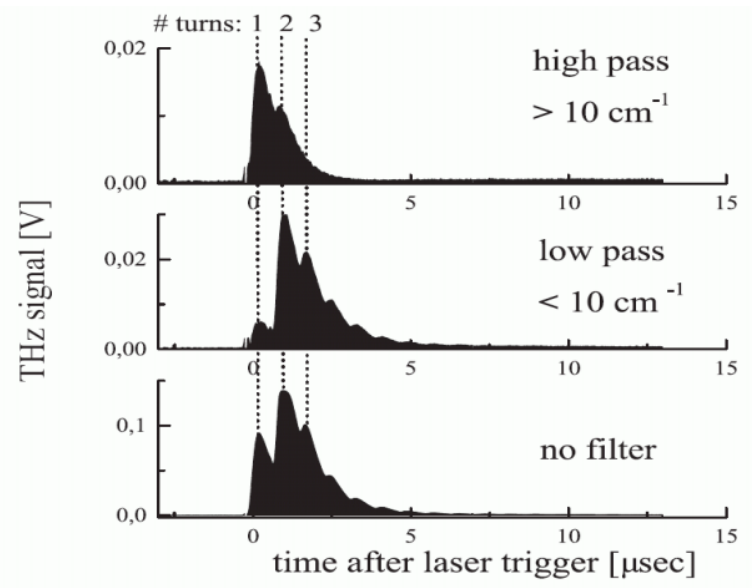

Figure 3: THz-"afterglow" time trace over several turns for different wavelength ranges of the radiation.

experimentally confirmed by the $\mathrm{THz}$ afterglow patterns in Fig.4. Here, the oscilloscope traces of the THz signal are plotted for different laser pulse widths measured with an autocorrelator. The minimum pulse length was $45 \mathrm{fs}$ fwhm. Using the stretcher within the amplifier the pulse length was varied by introducing a "chirp" to the pulse i.e. the pulse gets a frequency that varies in time. At a pulse length

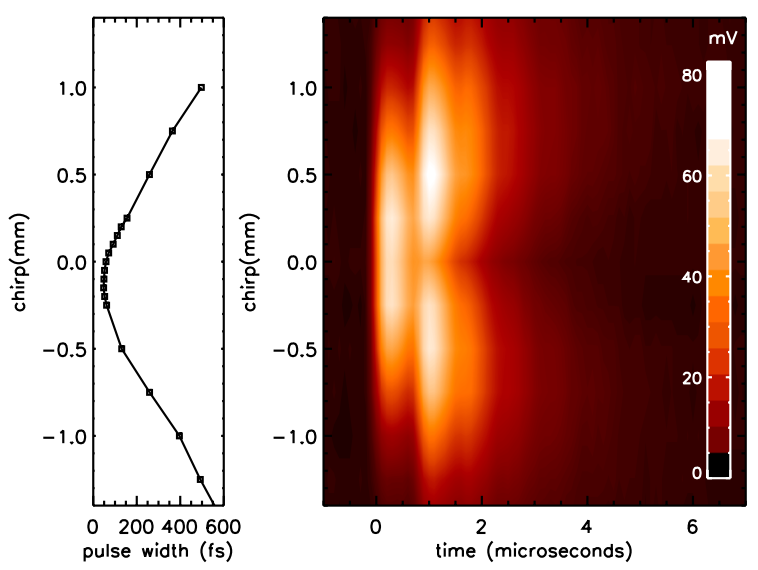

Figure 4: Colour coded plot of the $\mathrm{THz}$ intensity versus time (right) while the laser pulse was chirped. The "chirp" was measured indirectly using the mm-scale at the stretcher versus pulse length at the autocorrelator (left).

of $75 \mathrm{fs}$ as measured on the laser table (negative chirp), the afterglow suddenly vanishes within $10 \mathrm{fs}$ in pulse length variation. Comparison with the measured fs X-ray intensity and scraper measurements [6] in fact confirm maximum energy modulation at this chirp. It pre-compensates dispersion of subsequent optical elements (e.g. vacuum window). Since the method yields a nondestructive shot-to-shot response that the laser pulse chirp is pre-compensated in the 
right way, it has been routinely used to optimize femtoslicing for fs x-ray experiments.

\section{Slice and bunch shapes}

Spectra were measured discriminating the first turn by a filter like in Fig. 3 employing a slow, but broadband $4.2 \mathrm{~K}$ Si-bolometer using a $600 \mathrm{~cm}^{-1}(18 \mathrm{THz})$ low pass filter and a Martin-Puplett spectrometer [10]. The beamline transmission is corrected by measuring the incoherent spectra. In order to reconstruct the electron density a reasonable assumption for the pulse shape in time domain using two Gaussians $G_{1}$ and $G_{2}$ was made, where the first is the broad one of length $\sigma_{1}$ of energy modulated electrons and the second, of short length $\sigma_{2}$, is a depletion of electrons:

$$
\rho(z)=1+G_{1}\left(z, \sigma_{1}\right)-G_{2}\left(z, \sigma_{2}\right)
$$

Using an iterative routine the two $\sigma$-values are varied in time domain for best fit in frequency domain to the measured power spectrum as plotted Fig.5a. The fit yields $\sigma_{2}=130 \mathrm{fs}$ and $\sigma_{1}=210 \mathrm{fs}$. Inserting these values into equation 2 the electron density distribution matches initial simulations in Fig.5b. It is evident that the THz source is not a


Figure 5: Pulse reconstruction of the longitudinal electron density using an iterative fit method applied to a measured $\mathrm{THz}$ spectrum (a). Comparison of the reconstruction and tracking simulations (b) in time domain.

complement of a short fs electron bunch. Note, that this is a structure in the dipole $12 \mathrm{~m}$ behind the radiator but the measurement supports the theoretical prediction of sub-100 fs pulse length for the fs x-ray pulses.

Using the afterglow as an indicator for the energy transfer, the patterns in Fig.2 remain unchanged in shape while detuning the longitudinal synchronization. In that case the longitudinal bunch shape is probed in time domain detecting the square root of the total $\mathrm{THz}$ signal unless bursting CSR is involved. Two examples for the two different user modes of BESSY II are given in Fig.6. Due to the higher electron density in compressed bunches down to $3 \mathrm{ps}$ (rms), $\mathrm{THz}$ detection of the energy modulation is possible even at $20 \mathrm{pC}$ bunch charge. A small phase jitter between bunch and laser appears as sidebands of the $\mathrm{THz}$ peak (and harmonics) on a spectrum analyzer if the delay is detuned onto the head- or tail slope of the bunch.

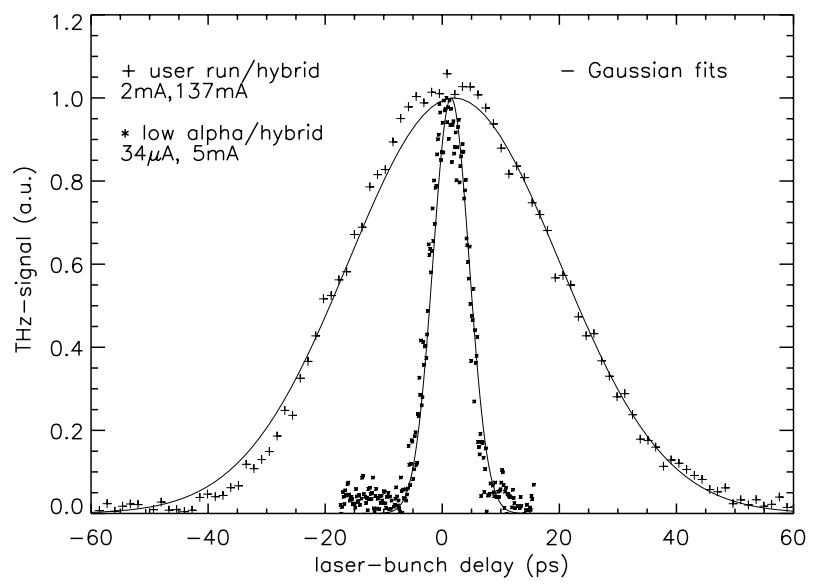

Figure 6: Normalized bunch shape measurements using the THz signal for two different operation modes at BESSY II.

\section{CONCLUSIONS}

The temporal and spectral characterization of coherent $\mathrm{THz}$ emission from a dispersed bunch slice after a femtosecond laser-electron interaction yields a detailed information for optimizing the interaction itself. A dedicated $\mathrm{THz}$ diagnostics for femtoslicing facilities and laser seeded FELs seems to be useful.

\section{ACKNOWLEDGEMENTS}

We are indebted to a very large number of BESSY staff members who are involved in the femtoslicing project. Special thanks to D. Ponwitz for the technical technical work at the $\mathrm{THz}$ beamline.

\section{REFERENCES}

[1] R.W. Schoenlein et al., Appl. Phys. 71, p. 1, 2000.

[2] K. Holldack, S. Khan, R. Mitzner, T. Quast, this conference.

[3] M. Abo-Bakr, J. Feikes, K. Holldack, H.-W. Hübers, G. Wüstefeld, Phys. Rev. Lett. 88, p. 2548011, 2002.

[4] K. Holldack et al., EPAC'04, Lucerne (2004), p. 834.

[5] F. Sannibale et al., EPAC'04, Lucerne (2004), p. 2448.

[6] K. Holldack, S. Khan, R. Mitzner, T. Quast, PRSTAB Vol.8(2005), p. 040704.

[7] S. Khan et al., PAC'03, Portland (2003), p. 836.

[8] K. Holldack et al., to be published.

[9] H. Wiedemann, "Particle Accelerator Physics I", Springer, Berlin (1998), p. 179.

[10] on loan from U. Schade (bolometer) and H.W. Hübers (spectrometer). 